\title{
Caracterización morfoagronómica de 25 clones de estevia (Stevia rebaudiana Bertoni.), en condiciones del valle del Sinú medio.
}

\section{Morphoagronomical characterization of 25 clones of stevia (Stevia rebaudiana Bertoni.), in conditions of the middle Sinú valley.}

\author{
Ana I. Pico-González ${ }^{1 *}$, Hermes Araméndiz-Tatis ${ }^{1 \oplus}$; Dairo J. Pérez-Polo ${ }^{1 \oplus}$ \\ Recibido para publicación: septiembre 10 de 2020- Aceptado para publicación: diciembre 27 de 2020
}

\begin{abstract}
RESUMEN
El cultivo de estevia es una actividad agrícola viable en Colombia y la región Caribe, que se fundamenta en el clon Morita II, representado un alto riesgo ante factores bióticos y abióticos. Por tal razón, la caracterización morfoagronómica de 25 clones de la colección de germoplasma en la Universidad de Córdoba, es importante con el fin de agruparlos de acuerdo a su afinidad genética e identificar y seleccionar los de mayores ventajas agronómicas. Para tal fin, se aplicaron 19 descriptores morfo-agronómicos (11 cuantitativos categorizados y ocho cualitativos); se realizó un dendograma mediante el método de Ward para la distinción entre grupos y un análisis multivariado de componentes principales para identificar los descriptores que más aportaron a la variabilidad. Se identificaron dos componentes principales, el primero, aportando un $47,74 \%$ y el segundo, un $21,67 \%$ de la variación total, soportado por caracteres relacionados con el rendimiento; así mismo, la identificación de seis clones de floración tardía, buena producción de hoja seca y hábito de crecimiento erecto, lo que resulta de interés para adelantar programas de mejoramiento genético en la especie.
\end{abstract}

Palabras clave: Análisis de componentes principales; Recurso fitogenético; Variabilidad genética.

\footnotetext{
${ }^{1}$ Universidad de Córdoba, (Facultad de Ciencias Agrícolas, departamento de Ingeniería Agronómica), Montería, Colombia. *Autor para correspondencia: Ana I. Pico Gonzalez Email:apicogonzalez@correo.unicordoba.edu.co
}

\begin{abstract}
Stevia cultivation is a viable agricultural activity in Colombia and the Caribbean region, which is based on the Morita II clone, representing a high risk of biotic and abiotic factors. For this reason, the morphoagronomic characterization of 25 clones from the germplasm collection at the University of Córdoba is important in order to group them according to their genetic affinity, and to identify and select those with the best agronomic advantages. For this purpose, 19 morphoagronomic descriptors were applied (11 quantitative and 8 qualitative); a dendrogram was performed using Ward's method to distinguish among groups and a multivariate analysis of principal components to identify the descriptors that contributed to variability. Two main components were identified, the first, contributing with $47,74 \%$ and the second with $21,67 \%$ of the total variation, supported by performance-related characters. Likewise, the identification of 6 late flowering clones, dry leaf production and erect growth habit, which is of interest to advance in genetic breeding programs in the species.
\end{abstract}

Keywords: Analysis of main components; Plant genetic resources; Genetic variability.

\section{Cómo citar}

Pico-González, A.I., Aramendiz-Tatis, H. y Pérez-Polo, D.J. 2020. Caracterización morfoagronómica de 25 clones de estevia (Stevia rebaudiana Bertoni.), en condiciones del valle del Sinú medio. Temas Agrarios 25(2):106-116.

https://doi.org/10.21897/rta.v25i2.2365 


\section{INTRODUCCIÓN}

Stevia rebaudiana Bertoni, es una planta originaria del sudeste de Paraguay, familia de las Asteráceas, conocida como "hoja dulce". Es un arbusto perenne que se utiliza para la producción comercial por un período de cinco o más años (Duran et al., 2012). Contiene en sus hojas, agentes edulcorantes naturales sin aporte calórico, siendo los primarios el esteviósido y los rebaudiósidos A y C (Xiang et al., 2019); es empleada para el consumo como sustituto de la sacarosa, con efectos positivos sobre la salud en los tratamientos contra el sobrepeso y diabetes al no incrementar los niveles de azúcar en la sangre, enfermedades que pueden conducir al desarrollo de múltiples complicaciones (Vaghela y Soni 2020).

Conocer la diversidad del germoplasma en las especies, es vital para el éxito de un programa de mejoramiento genético. Poblaciones con variabilidad genética limitada, representan una amenaza para la evolución de una especie por su vulnerabilidad a factores bióticos y abióticos. Así mismo, para lograr avances importantes en el mejoramiento genético en poblaciones con caracteres de interés, pues el efecto fundador es una limitante en el progreso genético, sobre características como rendimiento de biomasa, arquitectura de la planta, contenido de esteviósidos, entre otros. Por lo tanto, la caracterización de los recursos fitogenéticos nos permite discriminar las accesiones y la disponibilidad de rasgos útiles en los bancos de genes (Tang et al., 2012; Egbadzor et al., 2014; Correa et al., 2019); lo cual constituye un paso importante para la identificación e hibridación de padres con miras a la obtención de progenies que expresen mejores atributos agronómicos (Othman et al., 2018; Cosson et al., 2019).

Reportes sobre caracterización en estevia realizados por Othman et al. (2018), en cultivares originarios de Malasia y Paraguay, revelaron la existencia de una amplia variabilidad genética entre las accesiones de estevia; de igual forma, Francisco et al. (2018), identificaron clones promisorios para el desarrollo de nuevas variedades de estevia con genotipos brasileños. Sin embargo, Kumari et al. (2018), recomiendan realizar más trabajos de caracterización en este cultivo, que permitan descubrir cultivares potenciales desde el punto de vista químico y agronómico en otros países.

El mercado actual demanda cultivares adaptados a condiciones tropicales, con rendimientos de biomasa de 4.100 a 7.000 kg. ha-1 (Parris et al., 2016) y de esteviósidos entre 720 a 1023 kg. ha-1 (Munz et al., 2018), lo cual surge ante la demanda de edulcorantes en el mercado mundial (Angelini et al., 2018; Mahajan y Pal 2021). Así mismo, cultivares que presenten floración tardía, erectos, con tolerancia a factores bióticos y abióticos, con esteviósidos de alta calidad fuera de los centros tradicionales de producción, principalmente rebaudiósido A (Parris et al., 2016) y una mayor relación rebaudiósido A/esteviósido, que defina la calidad de la hoja (Hernández et al., 2016).

Por tanto, el objetivo de la presente investigación consistió en caracterizar morfoagronómicamente 25 clones de estevia seleccionados bajo condiciones del valle del Sinú medio, con el fin de agruparlos de acuerdo con su afinidad genética e identificar y seleccionar los de mayores ventajas agronómicas.

\section{MATERIALES Y MÉTODOS}

El experimento se realizó en el año 2017 en las instalaciones de la Facultad de Ciencias Agrícolas de la Universidad de Córdoba, localizada en las coordenadas geográficas $8^{\circ} 47^{\prime \prime}$ de Latitud N y a los $75^{\circ} 51^{\prime \prime}$ de Longitud W con respecto al meridiano de Grengwich, con precipitación promedio de 1141,6 mm, humedad relativa del $79 \%$, temperatura promedio anual de $28,3^{\circ} \mathrm{C}$ (IDEAM 2017) y 
zona de vida denominada bosque seco tropical (bs-T), según la clasificación de Holdrige (Palencia et al., 2006).

El establecimiento en campo se realizó en febrero del año 2017, en un área de 116,3 $\mathrm{m}^{2}$, bajo un diseño completamente al azar, con 25 tratamientos. La distancia entre plantas fue de 0,20 m y entre surco 0,40 m, para una

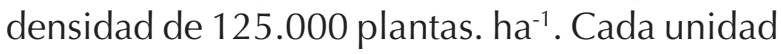
experimental estuvo conformada por dos surcos, con ocho plantas en cada surco para un total de 16 plantas por tratamiento, distribuidas al azar, conformando así 400 plantas efectivas y 96 de borde, utilizando el cultivar Morita II.

Las prácticas culturales se realizaron considerando las indicaciones de Jarma et al. (2005), se estableció un sistema de protección con polisombra a una altura de $2,5 \mathrm{~m}$, para un nivel de radiación incidente del $46 \%$ (promedio de radiación 320,3 $\mu$ moles fotones. $\mathrm{m}^{-2} \cdot \mathrm{s}^{-1}$ ). Posteriormente, se hizo un ahoyado a $10 \mathrm{~cm}$ de profundidad, el cual se rellenó antes del trasplante con lombriabono (40g), compost $(\mathrm{pH}$ de 7,9 y conductividad de $0,60 \mathrm{dS} / \mathrm{m}$ ) y arena en mezcla (223g). De igual manera, se realizó fertilización foliar cada 15 días con elementos menores con una dosis de $2 \mathrm{cc} /$ litro de agua y NPK de forma foliar en dosis de 4 cc/litro de agua.

El control de arvenses se realizó previo al establecimiento del experimento, con la aplicación de la mezcla de 200cc del ingrediente activo Glifosato+100cc de Diuron, en $20 \mathrm{~L}$ de agua antes del establecimiento del cultivo en campo.

El manejo de problemas fitosanitarios, para el caso de ácaros (Tetranychus urticae) y Thrips (Thrips stevi) con Abamectina en dosis de 2 cc/litro agua y enfermedades foliares con Azoxistrobin y Difenoconazol, a razón de 2cc/ litro de agua.
Las variables de respuesta fueron ocho cualitativas y once cuantitativas, considerando los descriptores propuestos por Araméndiz (2014), por ser más representativos para discriminar germoplasma de estevia. Para la toma de información de las variables cualitativas, éstas se realizaron sobre la base de diez plantas, atendiendo los criterios de guía propuestos por el mismo autor y que correspondieron a pubescencia del tallo, forma de la hoja, pubescencia de la hoja, color de la hoja, borde de la hoja, capacidad de rebrote, color de la flor y hábito de crecimiento. Las evaluaciones se realizaron mediante observación visual previo a la realización de la cosecha, se detectaron las variaciones morfológicas ocurridas en las plantas de los diferentes clones. Se empleó estadística descriptiva para el cálculo de la frecuencia de aparición (\%) de las variantes evaluadas para cada atributo cualitativo estudiad y se determinó la moda para cada variable.

Adicionalmente, fueron medidos once caracteres cuantitativos considerando cinco plantas en competencia y tomadas al azar en cada unidad experimental, los datos fueron categorizados y corresponden a número de tallos verticales y número de nudos del tallo principal, a través de conteo; diámetro del tallo, con la ayuda de un nonio o pie de rey, expresado en cm; longitud de los entrenudos, longitud y ancho de la hoja, con la ayuda de una regla indicada en $\mathrm{cm}$; número de brotes laterales primarios y secundarios, a través de conteo; días a floración, en días con la emisión del primer botón floral; altura de la planta, desde la base del tallo hasta el ápice del tallo principal antes de la cosecha, en $\mathrm{cm}$ y rendimiento de hoja seca, expresado en $\mathrm{kg} \cdot \mathrm{ha}^{-1}$, se realizó el corte de la planta manteniendo tres pares de hojas, y se obtuvo el peso fresco y peso seco en gramos, con una balanza analítica marca Precisa modelo BJ410C. Para la determinación del peso seco, se llevaron las muestras a estufa de secado marca DiEs en el laboratorio, durante 72 horas, a $70^{\circ} \mathrm{C}$ (De Lima et al., 2004). 
Para el estudio de estas variables cuantitativas, se realizó un análisis descriptivo considerando el valor de la media, el valor mínimo, el valor máximo, la desviación estándar y los coeficientes de variación. De igual manera, se realizó un dendograma mediante el método de agrupamiento jerárquico de Ward, que permitió apreciar gráficamente las afinidades entre genotipos y a su vez, un Análisis de Componentes Principales (ACP). Todos los datos se procesaron en el programa estadístico $R$ versión 3.3.2 (R 2015).

\section{RESULTADOS Y DISCUSIÓN}

\section{Análisis de caracteres cualitativos}

Desde el punto de vista morfológico, los clones acusaron variación en la pubescencia del tallo, forma de la hoja, color de la hoja, capacidad de rebrote y hábito de crecimiento, más no en las características asociadas con la pubescencia de la hoja, borde de la hoja y color de la flor. Las características con mayor polimorfismo corresponden a la forma de hoja, donde la modalidad lanceolada, registró un $48 \%$, seguida de la forma elíptica con $40 \%$ y ovalada con un $12 \%$ y hábito de crecimiento, donde el $60 \%$ de los clones acusaron hábito erecto, seguido de un $24 \%$ del hábito postrado y un $16 \%$ del hábito semierecto (Tabla 1 ).

La pubescencia del tallo presentó un $48 \%$ de tallos glabros y $52 \%$ de tallos pubescentes; el color de la hoja predominante fue verde oscuro, con un $68 \%$, seguido del verde medio, con un $32 \%$ y en la capacidad de rebrote, se observó que un $96 \%$ de los clones con la capacidad de hacerlo y un $4 \%$ de no hacerlo (Tabla 1 ).

De acuerdo con los resultados consignados para estas características, existe variabilidad genética muy útil, para identificar clones con características agronómicas deseables, como lo reportaron Othman et al. (2018) y Clemente et al. (2021), lo cual es resultado del proceso evolutivo, sobresaliendo la presencia de clones de hábito de crecimiento erecto, con capacidad de rebrote, hojas lanceolada, que permite una mayor eficiencia fotosintética, por la captura de altos niveles de radiación solar, que favorece un mayor crecimiento de la planta, e incidiendo en el rendimiento de hoja seca (Munz et al., 2018), dado que una alta relación hoja-tallo, es deseable para una mayor producción de esteviósidos (Hernández et al., 2016; Francisco et al., 2018) y reducción de la humedad en la parte basal con menores riesgos de enfermedades foliares.

El hecho de presentar buena capacidad de rebrote, favorece la realización de varias cosechas, y la presencia de tallo y hojas glabras, es ideal por su contenido de aceites esenciales como fitol, nerolidol y spathulenol (Benelli et al., 2020), actuando como insecticidas efectivos y ecológicos contra pulgones, dado que éstos pueden causar daño en las hojas, lo que sería muy significativo al causar mermas en la producción de biomasa. Resultados semejantes en la caracterización de germoplasma, fueron obtenidos por Tateo et al.(1998), y corrobora lo expresado por Singh et al. (2017), quienes destacaron que los clones obtenidos a través del uso de semilla sexual generaron variabilidad genética que pudo ser aprovechada en el mejoramiento genético.

Las características pubescencia de las hojas, borde de la hoja y color de la flor, fueron 100\% presente en los clones, siendo glabras, dentada y color blanco y, por lo tanto, carentes de evolución en la población estudiada.

\section{Análisis de caracteres cuantitativos}

Los valores de la estadística descriptiva para cada característica están consignados en la tabla 2, donde el número de tallos verticales y rendimiento de hoja seca, presentaron los mayores coeficientes de variación, por acusar registros superiores al $60 \%$, con valores 
promedios de 1,56 tallos verticales y $467 \mathrm{~kg}$. $\mathrm{ha}^{-1}$; en tanto que los coeficientes de variación menor al 20\%, están asociados a la longitud de los entrenudos, longitud de la hoja y días a floración, con valores promedios de 0,81 cm, 4,67cm y 71 días a floración. Las demás características presentaron variación entre $27,16 \%$ y $43,87 \%$.
De acuerdo con los resultados obtenidos, la variación genética observada entre dichos clones (Othman et al., 2018) posibilita identificar y seleccionar genotipos deseados para llenar las expectativas de productores, al presentar días floración igual o superior a 95 días, favorables valores en número de tallos verticales, longitud y ancho de la hoja,

Tabla 1. Modalidades, frecuencia y porcentaje de características cualitativas, evaluadas en 25 clones de Stevia rebaudiana (Bertoni), en Montería-Córdoba.

\begin{tabular}{llcc}
\hline \multicolumn{1}{c}{ Característica } & Modalidad & Frecuencia & Porcentaje \\
\hline Pubescencia del tallo & Glabro & 12 & 48 \\
Forma de la hoja & Pubescente & 13 & 52 \\
& Lanceolada & 12 & 48 \\
& Elíptica & 10 & 40 \\
Pubescencia de la hoja & Ovalada & 3 & 12 \\
Color de la hoja & Glabra & 25 & 100 \\
& Pubescente & 0 & 0 \\
Borde de la hoja & Verde claro & 0 & 0 \\
Capacidad de rebrote & Verde medio & 8 & 32 \\
& Verde oscuro & 17 & 68 \\
Color de la flor & Liso & 0 & 0 \\
& Dentado & 25 & 100 \\
Hábito de crecimiento & Sí & 24 & 96 \\
& No & 1 & 4 \\
& Blanca & 25 & 100 \\
& Otro & 0 & 0 \\
\hline \multirow{3}{*}{} & Erecto & 15 & 60 \\
\hline
\end{tabular}

considerando que las características de la hoja, acusan una correlación positiva con el contenido de esteviósido y rebaudiósido A de acuerdo con Singh et al. (2017), puesto que un ciclo vegetativo más prolongado, permite una mayor altura de la planta, acumulando mayor cantidad de hojas debido a la presencia de más brotes laterales primarios y secundarios (Srivastava 2014; Othman et al., 2018) por ende de esteviósidos, mejorando así la relación rebaudiósido A/esteviósido, que define la calidad de la hoja (Hernández et al., 2016), este parámetro también se ve influenciado por la oferta ambiental, puesto que, los resultados obtenidos por Rachmawati y Asiyah (2017), indican que los niveles de esteviósido incrementan conforme la intensidad lumínica decrece. 
Tabla 2. Estadistica descriptiva para caracteres cuantitativos, evaluadas en 25 clones de Stevia rebaudiana (Bertoni), en Montería - Córdoba.

\begin{tabular}{lcccccc}
\hline Parámetro & Muestra & Promedio & Mínimo & Máximo & D. Estándar & CV (\%) \\
\hline NTV & 25 & 1,56 & 1,00 & 5,00 & 1,00 & 64,10 \\
NNTP & 25 & 6,52 & 3,00 & 14,00 & 2,86 & 43,87 \\
DT & 25 & 0,58 & 0,18 & 0,90 & 0,22 & 37,93 \\
LE & 25 & 0,81 & 0,72 & 0,95 & 0,06 & 7,41 \\
LH & 25 & 4,67 & 3,10 & 6,00 & 0,84 & 17,99 \\
AH & 25 & 1,62 & 1,20 & 3,00 & 0,44 & 27,16 \\
NBLP & 25 & 8,80 & 2,00 & 17,00 & 3,55 & 40,34 \\
NBLS & 25 & 9,36 & 3,00 & 15,00 & 3,63 & 38,78 \\
DF & 25 & 71 & 65,00 & 95,00 & 10,17 & 14,30 \\
AP & 25 & 20,60 & 15,40 & 38,60 & 5,61 & 27,23 \\
RDTO & 25 & 467,40 & 140,76 & 1123,04 & 326,70 & 69,90 \\
\hline
\end{tabular}

NTV=número de tallos vertical; NNTP: número de nudos del tallo principal; DT: diámetro del tallo; LE: longitud de entrenudos; LH: longitud de la hoja; AH: ancho de la hoja; NBLP: número de brotes laterales primarios; NBLS: número de brotes laterales secundarios $\mathrm{DF}=$ días a floración; $\mathrm{AP}=$ altura de planta a inicio de floración; RDTO= rendimiento de hoja seca por hectárea.

Considerando que el rendimiento de hoja seca es importante con respecto a la síntesis de esteviósidos y rebaudiósido A, los rendimientos de los clones oscilaron entre 140,76 a 1123,04 $\mathrm{kg}$. ha ${ }^{-1}$, muy inferiores a los de Francisco et al. (2018), quienes reportaron valores entre 2,8 y 4,0 t. ha ${ }^{-1}$, para la primera cosecha, esta diferencia se sustenta en carecer de días largos $(>11,30$ horas) y alta intensidad solar, que facilita un mayor período vegetativo, ya que cuando la planta inicia la floración, los fotoasimilados se acumulan en la flor y declina la fase vegetativa (Guerrero et al., 2015; Clemente et al., 2021).

\section{Análisis de componentes principales (ACP)}

El ACP consignado en la tabla 3, registró dos componentes con valor propio superior a 1,0 resultados que concuerdan con los reportados por Adewale et al. (2010); Terzic et al. (2012);
Gerrano et al. (2015), acumulando una varianza total del $69,4 \%$.

El primer componente explicó el 47,74\% de la variación total, muy cercano al 50\% y obedece principalmente a variables asociadas con el rendimiento, en su orden de contribución están el número de días a floración, rendimiento de hoja seca y número de brotes laterales primarios, resultados similares a los obtenidos por Abdelsalam et al. (2016), y son variables que como lo indican Clemente et al. (2021), tienden a incrementar desde el punto de máximo crecimiento vegetativo, hasta el inicio de floración. El segundo componente, explicó el $21,67 \%$ de la variación total, sustentado por el aporte de la altura de la planta al inicio de floración y rendimiento de hoja seca, que se contrapone principalmente con el número de nudos del tallo principal y diámetro del tallo. 
Tabla 3. Análisis de componentes principales para siete características agronómicas categorizadas en 25 clones de S. rebaudiana Bert.

\begin{tabular}{lcc}
\hline \multicolumn{1}{c}{ Característica } & Componente $\mathbf{1}$ & Componente 2 \\
\hline NNTP & $-0,251$ & $-0,492$ \\
DT & $-0,312$ & $-0,345$ \\
NBLP & $-0,425$ & - \\
NBLS & $-0,367$ & $-0,241$ \\
DF & $-0,499$ & $-0,104$ \\
AP & $-0,229$ & 0,636 \\
RDTO & $-0,472$ & 0,396 \\
Valor propio & 1,82 & 1,23 \\
\% Varianza & 47,74 & 21,67 \\
\% Var. Acumulada & 47,74 & $\mathbf{6 9 , 4 1}$ \\
\hline
\end{tabular}

NNTP: número de nudos del tallo principal; DT: diámetro del tallo; NBLP: número de brotes laterales primarios; NBLS: número de brotes laterales secundarios; DF: días a floración después del trasplante; AP: altura de planta al inicio de floración; RDTO: rendimiento de hoja seca por hectárea.

\section{Clasificación jerárquica}

El agrupamiento de los genotipos obtenido mediante las características fenotípicas por el método de agrupamiento jerárquico de Ward, señaló dos grupos cuya separación se dio a una distancia de 12 (Figura 1).

Un primer grupo, estuvo conformado por ocho clones (C16, C19, C5, C7, C4, C2, C13 y C18), destacándose los clones C4, C16, C19, C13 y
C18, cuya floración fue tardía y se les realizó una sola cosecha, a diferencia del resto de genotipos a los cuales se les realizaron dos cosechas. El grupo número dos por su parte, estuvo conformado por 17 clones, que corresponden a los genotipos C6, C21, C1, C8, C9, C22, C25, C17, C3, C10, C24, C15, C14, C20, C11, C12 y C23, clúster más grande y disperso que el grupo anterior, caracterizándose por estar constituido por cinco pequeños subgrupos.

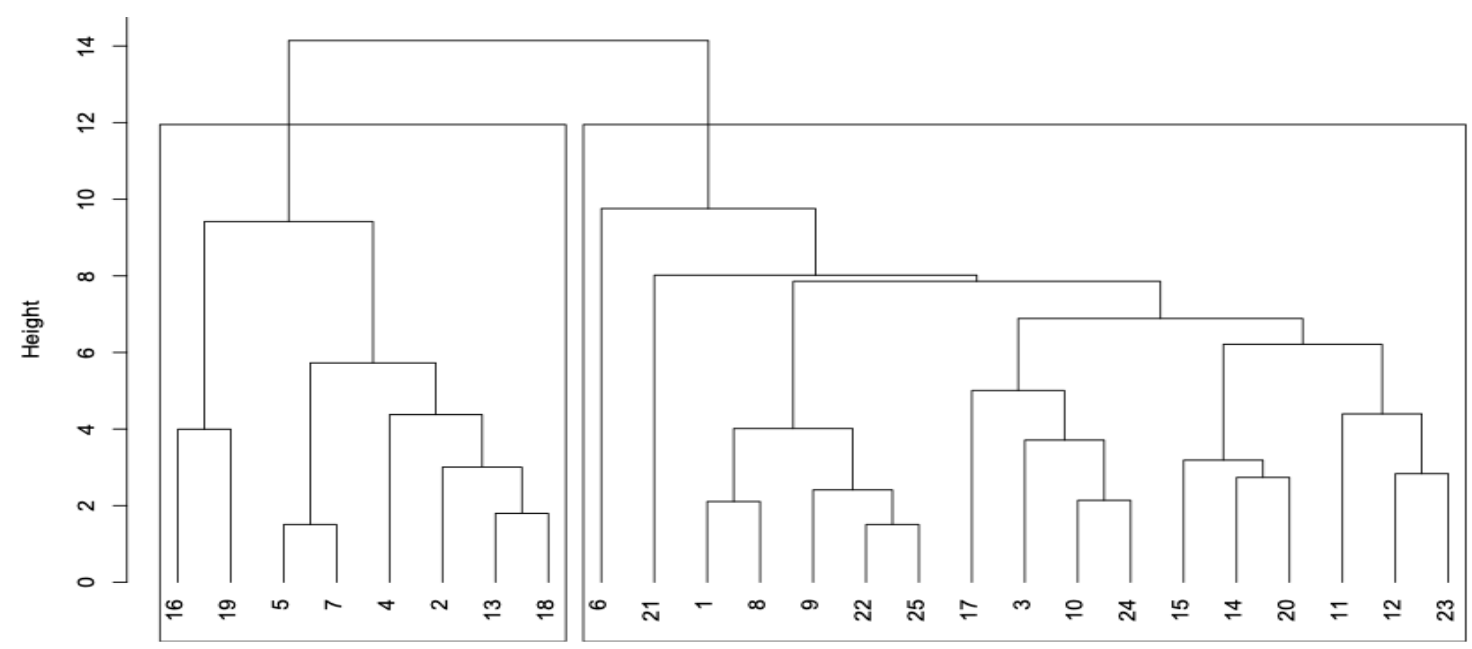

Figura 1. Dendograma para las características cualitativas y cuantitativas categorizadas de 25 clones de estevia. 
El primer grupo en mención se caracterizó por ser mucho más tardío en cuanto a floración, favoreciendo la síntesis y acumulación de esteviósidos (Yadav et al., 2011), mayor rendimiento de hoja, área foliar, intercepción de luz, $\mathrm{CO}_{2}$ y fotosíntesis. De esta forma, plantas con hojas más largas que anchas, y arquitectura erecta son deseables, pues presentarán un mayor índice de área foliar (Hastoy et al., 2019). Las plantas que crecen bajo condiciones protegidas presentan mayor capacidad de acumular más cantidad de biomasa sobre hojas y tallos, probablemente por niveles bajos de radiación y temperatura incidente (Jarma et al., 2020). De igual forma, se ha reportado que la intercepción de luz se relaciona también con la arquitectura y la densidad de plantas por hectárea (Munz et al., 2018).

Por otro lado, el grupo número dos sobresale, por su hábito de crecimiento erecto, presencia de 1 a 4 tallos verticales, hoja glabra, borde de la hoja dentado, caracteres similares fueron usados por Huber y Wehner (2021), para identificar cultivares superiores y ser usados en un programa de mejoramiento genético.

El hecho de tener un hábito de crecimiento erecto y tallos verticales es un aspecto agronómico favorable, primero, porque facilita la cosecha mecánica; toda vez que esta se realiza manualmente para los cultivares comerciales de hábito semipostrado y segundo, por representar un menor riesgo por contacto de las hojas con el suelo que favorecen la presencia de patógenos como Septoria sp., Fusarium sp., y Sclerotium sp., que afectan raíces y hojas (Hastoy 2018), obligando a la aplicación de fungicidas, reduciendo así los costos de producción. Debnath et al. (2019), señalaron que incrementos en la altura de planta y longitud entre nudos, son indicadores de la tolerancia de los genotipos a varios tipos de estrés; precisamente, cultivares con mayor resistencia y resiliencia a disturbios ambientales, son objetivos del mejoramiento en estevia (Al-Taweel et al., 2021).

\section{CONCLUSIONES}

Existe variabilidad genética en los clones estudiados, que puede ser usada directamente en la selección de clones para ser utilizados como padres para la incorporación de características agronómicas de interés, tal es el caso de floración tardía, que permite una mayor producción de biomasa seca de hojas y el hábito de crecimiento erecto, que facilitan la cosecha mecánica.

\section{Conflicto de intereses}

Los autores declaran que es un trabajo original y no existió conflicto de intereses de ningún tipo en la elaboración y publicación del manuscrito.

\section{REFERENCIAS}

Abdelsalam, N., Haraz, A., Khalid, A., Saleh, M. y Elsheikh, A. 2016. Genetic Improvement through Selection of Different Stevia rebaudiana Genotypes. Alexandria Science Exchange Journal 37(1): 10-25.

Doi: 10.21608/asejaiqjsae.2016.1902

Adewale, B., Okonj, C., Oyekanmi, A., Akintobi, D. y Aremu, C. 2010. Genotypic variability and stability of some grain yield components of cowpea. Afr J Agric Res 5(9): 874-880.

Doi: $10.5897 / A J A R 09.481$

Al-Taweel, S., Azzam, C., Khaled, K. y AbdelAziz, R. 2021. Improvement of stevia (Stevia rebaudiana Bertoni) and steviol glycoside through traditional breeding and biotechnological approaches. Journal of Breeding and Genetics 53(1): 88-111 https://tinyurl.com/83jfv4wv

Angelini L., Martini A., Passera, B. y Tavarini, S. 2018. Cultivo de Stevia rebaudiana Bertoni and Associated Challenges. In: Mérillon JM., Ramawat K. (eds) Sweeteners. Reference Series in Phytochemistry. Springer, Cham.

Doi: $\underline{10.1007 / 978-3-319-27027-28}$ 
Araméndiz, H. 2014. Descriptores para estevia (Stevia rebaudiana Bert.). Mimografiado. Universidad de Córdoba. 4p.

Benelli, G., Pavela, R., Drenaggi, E., Maggi, F. and Desneux, N. 2020. Phytol, (E)nerolidol and spathulenol from Stevia rebaudiana leaf essential oil as effective and eco-friendly botanical insecticides against metopolophium dirhodum. Industrial Crops and Products 155(11).

Doi: 10.1016/j.indcrop.2020.112844

Clemente, C., Angelini, L., Ascrizzi, R. and Tavarini, S. 2021. Stevia rebaudiana (Bertoni) as a multifunctional and sustainable crop for the mediterranean climate. Agriculture 11(123): 1-17.

Doi: 10.3390/agriculture11020123

Correa, E., León, R., Lobato, M., García, M., Muñoz, C. y Araméndiz, H. 2019. Caracterización morfoagronómica de la colección de germoplasma de ají dulce (Capsicum spp.) del Caribe colombiano. Revista Temas Agrarios 24(2): 81-95

Doi: $\underline{10.21897 / \text { rta.v24i2.1998 }}$

Cosson, P., Hastoy, C., Errazzu, L., Budeguer, C., Boutié, P., Rolin, D. and SchurdiLevraud1, V. 2019. Genetic diversity and population structure of the sweet leaf herb Stevia rebaudiana B., cultivated and landraces germplasm assessed by ESTSSRs genotyping and steviol glycosides phenotyping. Revista Plant Biology 19 (436): 1-11.

Doi: $10.1186 / s 12870-019-2061-y$

De Lima, O., Candeira, A. y Martínez, Z. 2004. Sistemas de Produção Estévia. Embrapa, Dourados, 43-46.

https://ainfo.cnptia.embrapa.br/digital/ bitstream/item/103385/1/SP5-2004.pdf

Debnath, M., Ashwath, N. and Midmore, D. 2019. Physiological and morphological responses to abiotic stresses in two cultivars of Stevia rebaudiana (Bert.) Bertoni. South African Journal of Botany 123(1): 124-132.

Doi: $\underline{10.1016 / j . s a j b .2019 .01 .025}$
Duran, S., Rodríguez, M., Cordón, K. and Record, J. 2012. Estevia (Stevia rebaudiana), edulcorante natural y no calórico. Revista Chil. Nutr. 39(4): 203206.

Doi:10.4067/S0717-5182012000400015

Egbadzor, K., Ofori, K., Yeboah, M., Aboagye, L., Opoku-Agyeman, M., Danquah, E. and Offei, S. 2014. Diversity in 113 cowpea (Vigna unguiculata (L.) Walp) accessions assessed with 458 SNP markers. Springerplus 3(541): 1-15. Doi: 10.1186/2193-1801-3-541

Francisco, F., Pinto, G., Pereira, M., Kanis, L. and Deschamps, C. 2018. Characterization of Stevia rebaudiana Bertoni accessions cultived in southern Brazil. Journal of Agricultural Science 10(3): 353-363

Doi: $\underline{10.5539 / j a s . v 10 n 3 p 353}$

Gerrano, A., Adebola, P., Rensburg, W. and Laurie, S. 2015. Genetic variability in cowpea (Vigna unguiculata (L.) Walp) genotypes. South African Journal of Plant and Soil 32(3): 165-174.

Doi: $10.1080 / 02571862.2015 .1014435$

Guerrero, D., Bär, A., Kryvenki, M. and Domínguez, M. 2015. Evaluación morfológica y fenológica de 10 clones de yerba dulce (Stevia rebaudiana) según el tiempo térmico. Revista Investig. Agrop. 41(3): 317-324.

http://www.redalyc.org/articulo. oa?id=86443147015

Hastoy, C., Cosson, P., Cavaignacc, S., Boutieb, P., Teguod, P., Rolina, D. and Schurdi, V. 2019. Deciphering performances of fifteen genotypes of Stevia rebaudiana in southwestern France through dry biomass and steviol glycoside evaluation. Revista Industrial Crops and Products 128(1): 607-619.

Doi: $\underline{10.1016 / \text { j.indcrop.2018.09.053 }}$ 
Hastoy, C. 2018. Caractérisation de la variabilité phénotypique de ressources génétiques de Stevia rebaudiana (Bertoni): analyse des composantes du rendement et critères de sélection en condition de production. Tesis doctoral, Sciences agricoles. Université de Bordeaux. Francia.

Hernández, J., Combatt, E., Jarma, A., Polo, J. y Rodríguez, L. 2016. Rendimiento y calidad de hojas de Stevia rebaudiana Bert. bajo la oferta edafológica y dos niveles de radiación en cinco regiones de Colombia. Revista U.D.C.A Actualidad \& Divulgación Científica 19(1): 77-85. https://revistas.udca.edu.co/index.php/ ruadc/article/view/112/1311

Huber, B. and Wehner, T. 2021. Performance of 16 Stevia rebaudiana seed cultigens for glycosides and yield in North Carolina. Revista Scientia Horticulturae 277(109803).

Doi: $\underline{10.1016 / j . s c i e n t a .2020 .109803}$

IDEAM (Instituto de Hidrología, Meteorología y Estudios Ambientales). 2017. Registros anuales de variables climatológicas, estación Turipaná-Cereté.

https://tinyurl.com/3vzfc6cw

Jarma, A., Combatt, E. and Jaraba, J. 2020. Growth and development of Stevia rebaudiana Bert., in high and low levels of radiation. Current Plant Biology 22(1): 1-5.

Doi: $\underline{10.1016 / j . c p b .2020 .100144}$

Jarma, A., Rengifo, T. y Araméndiz, H. 2005. Aspectos fisiológicos de estevia (Stevia rebaudiana Bertoni) en el Caribe colombiano: I. Efecto de la radiación incidente sobre el área foliar y la distribución de biomasa. Agronomía Colombiana 23(2): 207-216.

http://www.scielo.org.co/pdf/agc/v23n2/ v23n2a03.pdf

Kumari, N., Raina, R. and Sharma, Y. 2018. Interpopulation variations in morphochemical characteristics of Stevia rebaudiana Bertoni. Turkish Journal of Botany 42(4): 491-501.

Doi: $\underline{10.3906 / \text { bot-1712-26 }}$
Mahajan, M. and Pal, P. 2021. Attaining higher biomass and steviol glycosides yields of Stevia rebaudiana through adjustment of plant population and nitrogen rate. Industrial Crops and Products 165(2).

Doi: $\underline{\text { 10.1016/j.indcrop.2021.113426 }}$

Munz, S., Präger, A., Merkt, N., Claupeina , W. and Graeff-Hönninger, S. 2018. Leaf area index, light interception, growth and steviol glycoside formation of Stevia rebaudiana Bertoni under field conditions in southwestern Germany. Industrial Crops and Products 111(1): 520-528.

Doi: 10.1016/j.indcrop.2017.11.021

Othman, H., Osman, M. and Zainuddin, Z. 2018. Genetic variabilities of Stevia rebaudiana Bertoni cultivated in Malaysia as revealed by morphological, chemical and molecular characterizations. Revista Agrivita Journal of Agricultural Science 40(2): 267-283.

Doi: $\underline{10.1016 / \text { j.indcrop.2017.11.021 }}$

Palencia, G., Mercado, T. y Combatt, E. 2006. Estudio Agroclimático del Departamento de Córdoba. Gráficas del Caribe. Montería. 17.

https://www.researchgate.net/ publication/333356934 Estudio Agroclimatico_del_Departamento_de Cordoba

Parris, C., Shock, C. and Qian, M. 2016. Dry leaf and steviol glycoside productivity of Stevia rebaudiana in the Western United States. HortScience 51(10): 1220-1227. Doi: $10.21273 /$ HORTSCI11149-16

Rachmawati, D. and Asiyah, I. 2017. Response and stevioside levels of stevia (Stevia rebaudiana Bertoni) grown at different light intensity and water availability. Journal Pak. J. Bot. 49(5): 1689-1695. https://tinyurl.com/nnfchnef

R Development Core Team. 2015. R: A language and environment for statistical computing. R Foundation for Statistical Computing, Vienna, Austria. Version. 3.2.2. http://www.r-project.org/ 
Singh, M., Saharan, V., Rajpurohit, D., Sen, Y., Sharma, A. and Joshi, A. 2017. Criteria for selection of superior Stevia rebaudiana plant for propagation establishment. Journal of Pharmacognosy and Phytochemistry 6(4): 1362-1365.

Doi: $\underline{10.13140 / R G .2 .2 .25023 .36005}$

Srivastava, S. 2014. Influence of water stress on morpho-physiological and biochemical aspects of medicinal plant Stevia rebaudiana. Life Sciences Leaflets 49: 3543.

https://tinyurl.com/dcyu5bpp

Tang, H., Tie, M., Luo, Q., Zhu, Y., Lai, J. and Li, H. 2012. A Review of Molecular Makers Applied in Cowpea (Vigna unguiculata (L.) Walp) (Review) Breeding. Journal of Life Sciences 6(11): 1190-1199.

https://tinyurl.com/2aekcbzb

Tateo, F., Mariotti, M., Bononi, M., Lubian, E., Martello, S. and Cornara, L. 1998. Stevioside content and morphological variability in a population of Stevia rebaudiana (Bertoni) Bertoni from Paraguay. Revista Italian Journal of Food Science 10(3): 261-267.
Terzic, S., Atlagic, J., Maksimovic, I., Zeremski, T., Zoric, M., Miklic, V. and Balalic, I. 2012. Genetic variability for concentrations of essential elements in tubers and leaves of Jerusalem artichoke (Helianthus tuberosus L.). Revista Scientia Horticulturae 36(1):135-144. Doi: $\underline{10.1016 / j . s c i e n t a .2012 .01 .016}$

Vaghela, S. and Soni, A. 2020. Comprehensive overview of Stevia rebaudiana and its secondary metabolite sweeteners. Türk Bilimsel Derlemeler Dergisi 13(2): 26138p.

https://tinyurl.com/3366apbz

Xiang, Z., Tang, X., Liu, W. and Song, Ch. 2019. A comparative morphological and transcriptomic study on autotetraploid Stevia rebaudiana (Bertoni) and its diploid. Plant Physiology and Biochemistry 143(1): 154-164.

Doi: 10.1016/j.plaphy.2019.09.003

Yadav, A., Singh, S., Dhyani, D. and Ahuja, P. 2011. A review on the improvement of Stevia (Stevia rebaudiana Bertoni). Canadian Journal of Plant Science 91(1): $1-27$.

Doi: $\underline{10.4141 / \text { cjps10086 }}$ 